\title{
Comunicación Interna en el área turística: el caso del Departamento de Lingüística Aplicada en la Universidad de Leuven (Bélgica)
}

\section{Internal Communication in the Touristic Sector: the Case of the Department of Applied Linguistics of the KU Leuven (Belgium)}

Dr. Kris Buyse - KU Leuven - kris.buyse@kuleuven.be

Dra. Almudena Barrientos-Báez - Escuela Universitaria de Turismo Iriarte (Universidad de La Laguna) almudenabarrientos@iriarteuniversidad.es

Dr. Francisco Javier Sánchez-Verdejo Pérez - Universidad de Castilla - La Mancha - FcoJavier.SVerdejo@uclm.es

Resumen: Los objetivos del curso de maestría en Comunicación Interna $(\mathrm{Cl})$ en el departamento de Lingüística Aplicada (LA) de la Universidad KU Leuven de Bélgica son que los estudiantes sepan producir o reescribir textos y discursos en los idiomas elegidos en registros especializados en el campo de la $\mathrm{Cl}$ en las empresas, y que logren tener un dominio del idioma extranjero elegido a nivel avanzado, especialmente para los registros especializados relacionados con la $\mathrm{Cl}$ en la industria turística.

El curso $\mathrm{Cl}$ potencia la manera de trabajar informes orales y escritos dentro de la empresa y los estudiantes se enfrentan a documentos españoles siempre que es posible (confidencialidad de datos). Sin embargo, en una encuesta realizada al final del curso, los estudiantes calificaron la autenticidad y el 
impacto motivador de los contenidos y el enfoque del curso como muy bajos, y las notas finales no alcanzaron los estándares generales de los cursos de maestría en el departamento de LA.

Por tanto, en las últimas dos ediciones del curso, se adoptó un enfoque de enseñanza de idiomas basado en tareas, con un fuerte énfasis en las tareas de la vida real, la creación de significado y la motivación bajo el paraguas del concepto del yo futuro (Future Self, Oyserman, Destin y Novin, 2015). En lugar de copiar textos y presentaciones de varias empresas españolas existentes, se pide a los estudiantes que inventen una nueva empresa con sede en España, cuyos departamentos estarán representados por pequeños grupos de estudiantes. Todas las tareas están destinadas a ser actividades significativas con tareas preliminares y posteriores, y su resultado debe publicarse en el blog público de la empresa. Se utilizan varias herramientas web 2.0 como Google Drive con el objetivo de fomentar el trabajo en equipo, la interacción y el pensamiento crítico dentro de la empresa turística.

Para medir el impacto del enfoque TBLT en la motivación de los estudiantes, así como la calidad de su producción, se realizó un análisis cuantitativo y cualitativo.

Palabras claves: Comunicación Interna; Estudiantes; Future Self; Turismo.

Abstract: The objectives of the Master's Degree in Internal Communication (Cl) in the Department of Applied Linguistics (LA) of the KU Leuven University of Belgium are that students know how to produce or rewrite texts and speeches in the languages chosen in specialized registers in the field of the $\mathrm{Cl}$ in the companies, and that they have a mastery of the foreign language chosen at an advanced level, especially for the specialized registries related to the $\mathrm{Cl}$ in the tourism industry. The $\mathrm{Cl}$ course enhances the way of working oral and written reports within the company and students face Spanish documents whenever possible (data confidentiality). However, in a survey conducted at the end of the 
course, students rated the authenticity and the motivating impact of the content and the approach of the course as very low, and the final grades did not reach the general standards of the master's courses in the department of LA. Therefore, in the last two editions of the course, a task-based approach to language teaching was adopted, with a strong emphasis on real-life tasks, the creation of meaning and motivation under the umbrella of the concept of the future self (Future Self, Oyserman, Destin \& Novin, 2015). Instead of copying texts and presentations from several existing Spanish companies, students are asked to invent a new company based in Spain, whose departments will be represented by small groups of students. All tasks are intended to be significant activities with preliminary and subsequent tasks, and their result should be published on the company's public blog. Several web 2.0 tools are used such as Google Drive with the aim of promoting teamwork, interaction and critical thinking within the tourism company. To measure the impact of the TBLT approach on student motivation, as well as the quality of its production, a quantitative and qualitative analysis was performed.

Keywords: Future Self; Internal Communication; Students; Tourism.

\section{Introducción}

Hoy muchas personas leemos y escribimos mucho más con pantalla y teclado que con folio o bolígrafo, una tendencia que ha hecho que en esta era digital leamos, escribamos, hablemos y escuchemos de otra manera, hasta llegar al punto de que a veces no se sabe si estamos escribiendo o hablando cuando practicamos géneros híbridos como el chat o la mensajería móvil. Aunque el auge de los medios de comunicación oral pueda invitar a imaginarnos una vida sin destrezas escritas, éstas son socialmente imprescindibles y siguen gozando, junto a las destrezas orales, de un alto prestigio en las sociedades civilizadas caracterizadas por nuevos tipos de 
literacidad (por ejemplo, la digital) y de géneros (típicamente híbridos y multimodales con rasgos verbales, visuales, auditivos, etc.).

Asimismo, en el contexto de una enseñanza de lenguas, se hace necesario basar la elección de los objetivos y materiales en los contextos comunicativos cambiantes, sin olvidar la necesidad irrenunciable (aunque su importancia depende del tipo de enseñanza) de preparar a los alumnos para la lectura y redacción de géneros más tradicionales.

Desde finales de los años 90, se impone una realidad docente en la que disminuyen las horas de contacto y aumenta el volumen de los conocimientos, pero sin que crezcan las capacidades de la memoria humana. Sin embargo, sí siguen aumentando las posibilidades técnicas. Gracias $-\mathrm{y}$ debido- a estas evoluciones, se valora cada vez más el trabajo autónomo y colectivo (electrónico o no, on y off line), tanto por parte de los alumnos — que se convierten cada vez más en usuarios - como por parte de los profesores ellos cada vez más coaches (tutores) de un autoaprendizaje asistido, además, para toda la vida-. Por otro lado, el crecimiento imparable de los conocimientos nos obliga a desplazar el centro de la atención de los conocimientos hacia las actitudes y destrezas necesarias para encontrar, evaluar, seleccionar y utilizar la información. Así, al alumno se le convierte en un individuo responsable que llega a comunicarse con soltura, que toma iniciativas y realiza proyectos tanto individualmente como en equipo (Buyse \& Torfs, 1999).

Paradójicamente, cada vez más, los profesores describen a sus alumnos como algo opuesto: se caracterizarían por mostrar falta de responsabilidad, autonomía e iniciativa, además de estar cada vez menos dispuestos a comunicarse, tanto oralmente como por escrito. Buyse (2011, p. 33): "in order to be able to use terminology correctly, today's students need to be motivated by triggers and rewards" (Gijselaers, 2007; estímulos y recompensas, traducción propia), whereas earlier student generations did not need them as they were trained to conscientiously and systematically process new (lexical 
and syntactic) structures and to incorporate them in their oral and written discourse. As a result, today's teachers should adapt their teaching to this new reality, integrating triggers and rewards into the learning process".

Son numerosos los estudios que muestran que en el aprendizaje no sólo intervienen aspectos meramente cognitivos sino factores personales, entre los que destaca la motivación (Dörnyei, 2005), que a su vez se desgrana, según Uribe, Gutiérrez y Madrid (2008) en componentes cognitivos, conativos, evaluativos y afectivos. El mismo aspecto afectivo favorece la adquisición de competencias cognitivas en el aprendizaje de la L2 (Saito, Dewaele, Abe y In'nami, 2018). Especialmente la motivación intrínseca -aparte de otras como la extrínseca, la instrumental y la integrativa- ha sido considerada esencial para despertar la motivación del alumno, como se desprende de numerosos estudios sobre la motivación (Boo, Dörnyei y Ryan, 2015). No obstante, desde que Dörnyei y Ushioda (2009) pusieron el enfoque en el sistema motivacional del yo, otros conceptos han tomado el relevo, como el ideal self (Markus \& Ruvolo, 1989), el possible self (Markus \& Nurius, 1986), el L2 self (Dörnyei, 2009) y el future self (Oyserman, Destin y Novin, 2015). Éste último ha sido adoptado por la Universidad KU Leuven (Bélgica) en su visión sobre la enseñanza como motor central para motivar al alumnado actual: "Possible or future selves are conceptions of ourselves in the future, including, at least to some degree, an experience of being an agent in a future situation (...).The university offers certain 'disciplines' or study programmes which students can choose from and which will have an academic impact on the identity (therefore: disciplinary future self). (...) students who are constantly reminded of their disciplinary future self, study in a deeper way. They perceive the learning environment in a positive way and achieve according to their capabilities better results" (KU Leuven, 2014, p. 35).

De ahí que éste haya sido también el concepto clave elegido para volver a motivar a los alumnos de un curso de maestría en Comunicación Interna (Cl) 
en el departamento de Lingüística Aplicada (LA) de la misma universidad, cuyos objetivos fueron, e.o., los siguientes al asumir la titularidad del curso:

- Los estudiantes saben producir o reescribir textos y discursos en los idiomas elegidos en registros especializados en el campo de la comunicación interna en las empresas.

- (...)

- Los estudiantes tienen un dominio de su idioma extranjero elegido en un nivel avanzado, especialmente para los registros especializados relacionados con la $\mathrm{Cl}$ en la industria turística.

El curso de $\mathrm{Cl}$ trata una serie de informes orales y escritos dentro de la empresa. Los estudiantes son confrontados con documentos españoles auténticos tanto como sea posible (lo que no siempre es viable para documentos de $\mathrm{Cl}$, debido a la naturaleza confidencial de estos documentos). Esto debería motivarlos y enriquecer al mismo tiempo su conocimiento del español económico real y representativo.

Sin embargo, en una encuesta realizada al final del curso, los estudiantes calificaron los contenidos y enfoques como poco auténticos y motivadores, y las calificaciones y resultados generales fueron inferiores a las normas generales de los cursos de maestría en el departamento de LA.

Por lo tanto, en las ediciones siguientes del curso, se adoptó un enfoque de Enseñanza de Lenguas Mediante Tareas (ELMT, o Task Based Language Teaching, véase Ellis, 2003), con un fuerte énfasis en tareas auténticas y significativas bajo el paraguas del concepto del Yo futuro (Future Self). En lugar de trabajar con materiales de compañías españolas existentes, se les pidió a los estudiantes que inventaran una nueva compañía con sede en España, cuyos departamentos estuvieran integrados por pequeños grupos de estudiantes, y en cuyo contexto realizaran tareas orales y escritas con actividades significativas cuyos resultados se publicaron en el blog público de la empresa. Se utilizaron varias herramientas típicas del mundo digital de los alumnos, como Google Drive, con el objetivo de fomentar el trabajo en equipo, 
la interacción y el pensamiento crítico. Tal como se prescribe en el ELMT, se trabajó para cada tarea con un plan de trabajo con tareas preliminares y posteriores (pre- and post tasks, véase Ellis 2003) que implican una serie de actividades (meta) cognitivas y motivadoras, es decir, agradables y realistas: "preferably a task also involves realworld processes of language use" (Ellis 2003, p. 9-10). Una tarea preliminar inspirada por el concepto del Yo futuro consistía por ejemplo en que el alumno se proyectara a sí mismo en el futuro laboral con el objetivo de imaginarse ejerciendo como un profesional en varias de sus funciones soñadas ( $y$ de las que al menos una la desempeñaría también en la empresa virtual), preguntándose qué actividades tendría que realizar en ese entorno y en qué medida necesitaría sus conocimientos del español.

Con el objetivo de medir el impacto de las intervenciones pedagógicas, en las encuestas al final de cada curso se volvió a medir los niveles de satisfacción de los alumnos, intentando conseguir así una respuesta objetiva a las siguientes preguntas de investigación (PI):

1) ¿En qué medida los alumnos evalúan las tareas como más motivadoras en comparación con sus compañeros de los cursos anteriores? (PI 1)

2) ¿En qué medida los alumnos evalúan las tareas como más auténticas en comparación con sus compañeros de los cursos anteriores? (PI 2)

3) ¿Cómo se calificó el output de las tareas en comparación con sus compañeros de los cursos anteriores? (PI 3)

Siguiendo a Vandekerckhove, Vandergraesen \& Cruysweegs (2009), se esperaba que los estudiantes con una motivación basada en un enfoque por tareas consideraran las tareas como experiencias de aprendizaje desafiantes y valiosas $\mathrm{y}$, por lo tanto, las realizaran de una manera más estructurada y exhaustiva. Bliuc y col. (2011), entre otros, afirman que los estudiantes a quienes se les recuerda constantemente su futuro disciplinario, estudian de manera más profunda, perciben el ambiente de aprendizaje de una manera positiva y logran de acuerdo con sus capacidades mejores resultados. 
Asimismo, con respecto a la profesión y anterior formación universitaria en Turismo en particular, Barrientos Báez (2019) afirma que hace falta invertir más en el desarrollo de las inteligencias múltiples y la inteligencia emocional para aumentar la motivación de los estudiantes durante su formación y de los profesionales durante su desempeño profesional.

Por lo tanto, nuestra hipótesis era que este enfoque implicara actividades significativamente más motivadoras, así como un mejor rendimiento.

\section{Método}

Para medir el impacto de las intervenciones basadas en el enfoque ELMT combinado con la pedagogía del Yo futuro en la motivación de los estudiantes (y en el maestro), así como en la calidad de su producción, se realizó un análisis cuantitativo y cualitativo: se hizo la misma encuesta que al final de la edición anterior para evaluar los niveles de motivación y autenticidad de todos los participantes, y se compararon las notas finales de los alumnos con las del año anterior.

Como el experimento basado en el aula $y$ las regulaciones departamentales no permitieron trabajar con grupos experimentales y de control, los resultados se compararon con los de la misma encuesta y las mismas actividades (pero sin el enfoque del ELMT y el del yo futuro) de las ediciones anteriores de curso.

Los participantes fueron 32 en total: 10 en el año de referencia (es decir, sin intervenciones pedagógicas nuevas), 12 en el primer año y 10 en el segundo después del año de referencia. Todos eran estudiantes neerlandófonos de Español como Lengua Extranjera (ELE) en el Máster de Comunicación Multilingüe (Máster de 1 año dentro de la formación de LA), con edades comprendidas entre 21 y 24 años. A pesar del carácter homogéneo de la población, se pidió a los participantes que entregaran, después de haber aceptado que sus respuestas fueran utilizadas en el marco de un proyecto de investigación, los siguientes metadatos: sexo, edad, nacionalidad, lengua(s) 
materna(s), lenguas extranjeras aprendidas, educación bilingüe, formación anterior, cursos de español anteriores, periodo(s) de inmersión en español. Las preguntas en la encuesta fueron validadas y las respuestas evaluadas por dos evaluadores independientes y especialistas en el tema de la motivación en ELMT (inter-rater reliability test for Kappa $=0.80$ con $p<0.001$ )). Todas las "preguntas", menos una que era de respuesta abierta, tenían la forma de afirmaciones que había que valorizar en una escala de Likert de 1 a 5 , donde "1" significaba "totalmente en desacuerdo" y " 5 " "totalmente de acuerdo". Las afirmaciones eran de uno de los siguientes tipos:

- "Las actividades fueron \{motivadoras | auténticas | suficientes | demasiadas | demasiado pocas | ..."

- "El feedback para cada actividad era suficiente | motivador | claro | ..."

- "Las actividades me permitieron tener una idea clara de cómo serán mis actividades profesionales en el futuro"

- "Los instrumentos utilizados fueron apropiados | motivadores | fáciles de usar | ..."

El análisis estadístico comprendió las siguientes pruebas:

- Estadísticas descriptivas: suma total, media, mediana, desviación estándar

- Pruebas T para comparar los grupos

- Prueba de correlación Spearman rank para relacionar los resultados con los metadatos

\section{Resultados}

La siguiente tabla contiene los valores medios de las notas finales y de una selección de ítems de la encuesta que permitan responder a las tres preguntas de investigación (PI). 
Tabla 1: Puntuaciones medias de las notas finales y de una selección de ítems de la encuesta.

\begin{tabular}{|c|c|c|c|c|c|c|c|c|c|c|c|}
\hline & 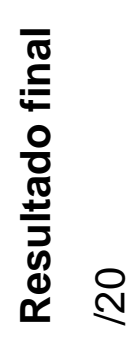 & 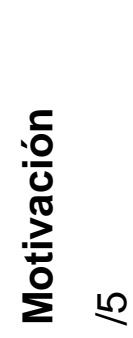 & 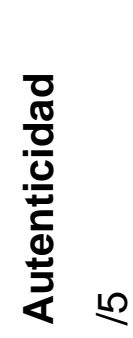 & 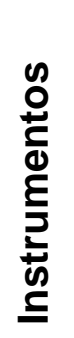 & 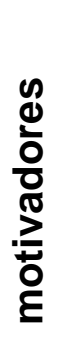 & 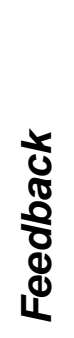 & 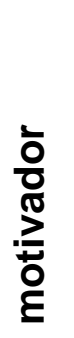 & 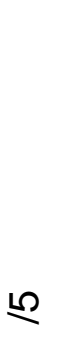 & $\begin{array}{l}\overline{0} \\
\frac{0}{0} \\
\frac{\pi}{\frac{\pi}{\pi}} \\
\frac{\pi}{0} \\
\frac{\pi}{0}\end{array}$ & $\begin{array}{l}0 \\
\frac{0}{2} \\
\frac{7}{2}\end{array}$ & $\begin{array}{l}\bar{\pi} \\
\frac{0}{0} \\
\frac{0}{9} \\
\frac{d}{0} \\
\frac{0}{2}\end{array}$ \\
\hline $\begin{array}{l}\text { Año } 0 \\
(\mathrm{~N}=10)\end{array}$ & 11.3 & 2.1 & 1.9 & 2.6 & & 3.5 & & & 1.9 & & \\
\hline $\begin{array}{l}\text { Año +1 } \\
(\mathrm{N}=12)\end{array}$ & 14.2 & 4.1 & 3.9 & 2.9 & & 4.1 & & & 3.9 & & \\
\hline $\begin{array}{l}\text { Año +2 } \\
(\mathrm{N}=10)\end{array}$ & 14.1 & 4.3 & 4.1 & 3.5 & & 4.0 & & & 4.0 & & \\
\hline
\end{tabular}

Fuente: Elaboración propia.

Con respecto a la PI1, se desprende que las actividades se han calificado como significativamente más motivadoras en los años "+1" y "+2" (es decir, los dos años en los que se hicieron las intervenciones pedagógicas descritas anteriormente) que en el año de referencia ("año 0"), es decir, que la media sube de 2.1 a 4.1 y 4.3 sobre 5 , respectivamente $(\mathrm{t}(18)=-6.35, \mathrm{p}=<.000$; $t(20)=-7.71, p=<.000)$. La desviación estándar varía entre 0.63 y 0.77 . No se pudo encontrar ninguna correlación significativa con los metadatos.

Respecto de la PI2, las actividades se han calificado significativamente más motivadoras en los años "+1" y "+2" (es decir, los dos años en los que se hicieron las intervenciones pedagógicas descritas anteriormente) que en el año de referencia ("año 0"), es decir, que la media sube de 2.1 a 4.1 y 4.3 sobre 5 , respectivamente $(\mathrm{t}(18)=-5.33, \mathrm{p}=<.000 ; \mathrm{t}(20)=-6.65, \mathrm{p}=<.000)$. La desviación estándar varía entre 0.72 y 0.77 . No se pudo encontrar ninguna correlación significativa con los metadatos. 
Con respecto a la PI3, las notas finales de los años "+1" y "+2" se mejoraron significativamente con respecto al "año 0 ", es decir: de 11.3 a 14.2 y 14.0 sobre 20 , respectivamente $(\mathrm{t}(18)=-3.40, \mathrm{p}=\langle 0.003 ; \mathrm{t}(20)=-3.17, \mathrm{p}=0.004)$. La desviación estándar varía entre 2.2 y 2.3. No se pudo encontrar ninguna correlación significativa con los metadatos.

Los otros ítems que también se encuestaron pero se quedaron fuera de las preguntas de investigación, es decir: sobre el carácter motivador del feedback y de las herramientas, así como sobre la creación de una idea clara sobre el futuro profesional, muestran evoluciones positivas parecidas desde el año de referencia a los años posteriores.

\section{Conclusiones}

Los estudiantes de las últimas ediciones mostraron un grado de motivación significativamente mayor en la encuesta y se desenvolvieron también significativamente mejor en las actividades, algo que se supone puede relacionarse con las intervenciones pedagógicas desde un enfoque de ELMT y basado en el concepto del yo futuro. Por tanto, concluimos que la nueva metodología se adapta mejor al curso, aunque se debe tener precaución ya que las poblaciones son muy homogéneas y tienen metadatos muy similares, pero no iguales, además de tratarse de grupos diferentes y consecutivos. En este sentido, la investigación se considera como un estudio piloto, esperando que en el futuro las regulaciones departamentales permitan estudiar grupos paralelos experimentales y de control.

\section{Bibliografía}

Barrientos, A. (2019). El desarrollo de la Inteligencia Emocional en los estudios del Grado en Turismo en la Universidad de La Laguna (Tenerife). Madrid: Universidad Camilo José Cela. Tesis doctoral. 
Boo, Z., Dörnyei, Z. \& Ryan, S. (2015). L2 motivation research 2005-2014: Understanding a publication surge and a changing landscape. System, $55,145-157$.

Buyse, K. (2011). Effective language learning in Isp contexts what most courses should do but fail to do. International Journal of Applied Linguistics, 161, 31-45.

Buyse, K. \& Torfs, G. (1999). Magister: El tutor electrónico de la Handelshogeschool. Un programa de "auto-estudio guiado" en un curso de español interactivo" en Enfoque comunicativo y Gramática. Santiago: Asele \& Universidad de Santiago de Compostela, 923-935.

Dörnyei, Z. (2005). The Psychology of the Language Learner Individual Differences in Second Language Acquisition. Nueva York: Routledge.

Dörnyei, Z. (2009). The L2 motivational self system. Motivation, Language Identity and the L2 Self. Eds. Z. Dörnyei and E. Ushioda. Bristol: Multilingual Matters, p. 9-42.

\& Murphey, T. (1983). Group Dynamics in the Language Classroom. Cambridge: Cambridge University Press.

Ellis, R. (2003). Task-based Language Learning and Teaching. Oxford: Oxford University Press.

Markus, H. \& Nurius, P. (1986). Possible Selves. American Psychologist, 41(9), 954-969.

\& Ruvolo, A. (1989). Possible Selves: Personalized representations of Goals. Goal Concepts in Personality and Social Psychology. Ed. L. A. Pervin. Hillsdale, NJ: Erlbaum, 211-241.

Gijselaers, W. (2007). Talking about My Generation. Keynote at EARLI 07: European Practice-based and Practitioner Research conference on 
Learning and Instruction for the new generation. Maastricht, 14-16 November 2007.

KU Leuven. (2014). Visie en beleidsplan onderwijs en studenten 2014-2017. Leuven: KU Leuven.

Oyserman, D., Destin, M. \& Novin, S. (2015). The context-sensitive future self: possible selves motivate in context, not otherwise. Self and ldentity, 14(2), 173-188.

Saito, K. Dewaele, J. M., Abe, M. \& In'nami, Y. (2018). Motivation, Emotion, Learning Experience and Second Language Comprehensibility Development in Classroom Settings: A Cross-Sectional and Longitudinal Study, Language Learning. A Journal of Research in Language Studies 68(3), 709-743.

Uribe, D., Gutiérrez, J. \& Madrid, D. (2008). Las actitudes del alumnado hacia el aprendizaje del inglés como idioma extranjero: estudio de una muestra en el sur de España. Porta Linguarum, 85-100.

Vandekerckhove, J., Vandergraesen F. \& Cruysweegs, B. (2009). Competent: een algemene didactiek in 101 lemma's. Wommelgem: Van In. 